\title{
Power Controlled Channel Allocation for Multiuser Multiband UWB Systems
}

\author{
W. Pam Siriwongpairat, Member, IEEE, Zhu Han, Member, IEEE, and K. J. Ray Liu, Fellow, IEEE
}

\begin{abstract}
Emerging ultra-wideband (UWB) technology offers a great potential for the design of high-speed short-range communications. However, in order for a UWB device to coexist with other devices, the transmitted power level of UWB is strictly limited by the FCC spectral mask. Such limitation poses a significant design challenge to any UWB system. An efficient management of the limited power is thus a key feature to fully exploit the advantages of UWB. In this paper, a cross layer multiuser multiband UWB scheme is proposed to obtain the optimal subband and power allocation strategy. Optimization criteria involve minimization of power consumption under the constraints on the packet error rate, the data rate, and the FCC limit. To ensure the system feasibility in variable channel conditions, an algorithm to jointly manage the rate assignment of UWB devices, subband allocation, and power control is proposed. A computationally inexpensive suboptimal approach is also developed to reduce the complexity of the problem, which is found to be $N P$ hard. Simulation results under UWB channel model specified in the IEEE 802.15.3a standard show that the proposed algorithm achieves comparable performances to those of the complex optimal full search approach, and it can save up to $61 \%$ of transmit power compared to the current multiband scheme in the standard proposal. Moreover, the proposed algorithm can obtain the feasible solutions adaptively when the initial system is not feasible for the rate requirements of the users.
\end{abstract}

Index Terms - Ultra-wideband (UWB), multiband orthogonal frequency-division multiplexing (OFDM), wireless personal area networks (WPANs), power control, channel allocation.

\section{INTRODUCTION}

$\mathbf{U}$ LTRA-WIDEBAND (UWB) is an emerging technology that offers great promises to satisfy the growing demand for low cost and high-speed digital wireless home networks. In 1998, the federal communications commission (FCC) has mandated that UWB radio transmission can legally operate in the range from $3.1 \mathrm{GHz}$ to $10.6 \mathrm{GHz}$, at a transmit power of $-41.3 \mathrm{dBm} / \mathrm{MHz}$ [1]. Traditional UWB technology is based on single-band systems [2]-[4] that directly modulate data into a sequence of pulses which occupy the available bandwidth

Manuscript received April 26, 2005; revised October 20, 2005 and April 13, 2006; accepted May 9, 2006. The associate editor coordinating the review of this paper and approving it for publication was W. Zhuang. This work was supported in part by MURI AFOSR under Cooperative Agreement F496200210217. This paper was presented in part at the IEEE Wireless Communications and Networking Conference, New Orleans, LA, Mar. 2005.

W. P. Siriwongpairat is with Meteor Communications Corporation, 22614 66th Ave. S., Kent, WA 98032, USA (email:PSiriwongpairat@meteorcomm.com).

Z. Han is with the Department of Electrical and Computer Engineering, Boise State University, Boise, ID 83725, USA (e-mail: hanzhu22@gmail.com).

K. J. R. Liu is with the Department of Electrical and Computer Engineering and Institute for Systems Research, University of Maryland, College Park, MD 20742, USA (e-mail: kjrliu@eng.umd.edu).

Digital Object Identifier 10.1109/TWC.2007.05298. of $7.5 \mathrm{GHz}$. Recently, innovative multiband UWB schemes were proposed in [5]-[7]. Instead of using the entire UWB frequency band to transmit information, multiband technique divides the spectrum into several subbands. Each subband occupies bandwidth of at least $500 \mathrm{MHz}$ in compliance with the FCC limit [1]. By interleaving the transmitted symbols across subbands, multiband UWB systems can still maintain the average transmit power as if the large $\mathrm{GHz}$ bandwidth is used. The advantage is that the information can be processed over much smaller bandwidth, thereby reducing overall design complexity, as well as improving spectral flexibility and worldwide compliance. The current leading proposal for the IEEE 802.15.3a wireless personal area networking (WPAN) standard [8] is based on multiband orthogonal frequency division multiplexing (OFDM), which utilizes a combination of OFDM and time-frequency interleaving [7]. The OFDM technique is efficient at collecting multipath energy in highly dispersive channels, as is the case for most UWB channels [7]. Time-frequency interleaving allows the OFDM symbols to be transmitted on different subbands. A frequency synthesizer can be utilized to perform frequency hopping. By using proper time-frequency codes, the multiband UWB system provides not only diversity, but also multiple access capability [7].

Since many applications enabled by UWB are expected to be in portable devices, low power consumption becomes a fundamental requirement. The low transmit power of UWB emissions ensures long life-time for the energy-limited devices. In addition, UWB systems are expected to support an integration of multimedia traffic, such as voice, image, data, and video streams. This requires a cross layer algorithm that is able to allocate the available resources to a variety of users with different service rates in an effective way. An overview of resource allocation in UWB communications is provided in [9]. In [10], the authors considered a joint rate and power assignment problem that is central in multiuser UWB networks, and proposed a radio resource sharing mechanism that performs a handshaking procedure to establish a communication link. In [11], the authors discussed a joint scheduling, routing, and power allocation problem with an objective to maximize the total utility of UWB system.

Most of the existing resource allocation schemes for UWB systems (see [9]-[11] and references therein) are based on single-band impulse radio technology. On the other hand, most research efforts on multiband UWB systems have been devoted to the physical layer issues [7], [12]-[13]. Some of the key issues in multiband UWB systems that remain largely unexplored are resource allocations such as power control and channel allocation. The current multiband proposal divides 
the subbands into groups, each comprising 2-3 subbands. A set of certain time-frequency codes is used to interleave the data within each band group [7]. This strategy lacks of the ability to allocate subbands optimally since the available subbands are not assigned to each user according to its channel condition. Moreover, in the multiband proposal [7], the transmit power of each user is equally distributed among its assigned subbands without any power adaptation to the channel variations. So adaptive optimization of the subband assignment and power control can greatly improve the system performances of multiband UWB systems.

In this paper, we propose a novel cross layer channel allocation scheme for multiband UWB wireless networks (e.g., a piconet, as in the IEEE 802.15.3 standard). By efficiently allocating the subbands, transmit power, and data rates among all users, the proposed scheme enables the multiband UWB system to operate at a low transmit power level, while still achieving desired performance. First, we formulate a subband assignment and power allocation problem as an optimization problem whose goal is to minimize the overall transmit power provided that all users achieve their requested data rates and desired packet error rate (PER), while the power spectral density complies with the FCC limit [1]. To take into account the fact that users in the multiband UWB system may have different data rates which in turn implies different channel coding rates, frequency spreading gains, and/or time spreading gains, our formulated problem considers not only the limitation on transmit power level, but also band hopping for users with different data rates. It turns out that the formulated problem is an integer programming problem whose complexity is $N P$ hard. Then, to reduce the complexity of the formulated problem, we propose a fast suboptimal algorithm that can guarantee to obtain a near optimal solution, but requires low computational complexity. In order to ensure the system feasibility in variable channel conditions, we further develop a joint rate assignment and power controlled channel allocation algorithm that is able to allocate resources to the users according to three different system optimization goals, namely maximizing overall rate, achieving proportional fairness, and reducing maximal rate. Simulation results based on UWB channel model specified in the IEEE 802.15.3a standard [14] show that the proposed algorithm achieves up to $61 \%$ of transmit power saving compared to standard multiband scheme [7]. Moreover, the proposed algorithm can also find feasible solutions adaptively when the initial system is not feasible for the rate requirements of the users.

The rest of the paper is organized as follows. Section II describes the system model of multiband UWB. In Section III, we first formulate the power controlled channel allocation problem. Then, a fast suboptimal scheme is developed. Finally, we propose a joint rate assignment and resource allocation algorithm to ensure system feasibility. Simulation results are given in Section IV and conclusions are drawn in Section V.

\section{SySTEM MODEL}

We consider a UWB system using multiband OFDM that has been proposed for the IEEE 802.15.3a WPAN standard [8]. As shown in Fig. 1, the available UWB spectrum, from 3.1 $\mathrm{GHz}$ to $10.6 \mathrm{GHz}$, is divided into $S$ subbands. Each subband
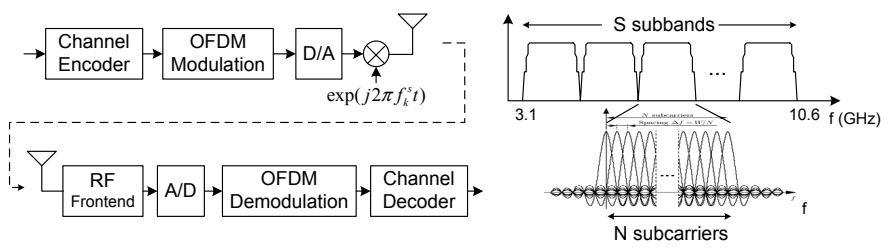

Fig. 1. Mulitband UWB system illustration.

TABLE I

RATE-DEPENDENT PARAMETERS

\begin{tabular}{|c|c|c|c|c|}
\hline $\begin{array}{c}\text { Data } \\
\text { Rate } \\
\text { (Mbps) }\end{array}$ & $\begin{array}{c}\text { Modu- } \\
\text { lation }\end{array}$ & $\begin{array}{c}\text { Coding } \\
\text { rate }\end{array}$ & $\begin{array}{c}\text { Conjugate } \\
\text { Symmetric } \\
\text { Inputs to IFFT }\end{array}$ & $\begin{array}{c}\text { Time } \\
\text { Spreading } \\
\text { Factor }\end{array}$ \\
\hline 53.3 & QPSK & $1 / 3$ & Yes & 2 \\
55 & QPSK & $11 / 32$ & Yes & 2 \\
80 & QPSK & $1 / 2$ & Yes & 2 \\
106.7 & QPSK & $1 / 3$ & No & 2 \\
110 & QPSK & $11 / 32$ & No & 2 \\
160 & QPSK & $1 / 2$ & No & 2 \\
200 & QPSK & $5 / 8$ & No & 2 \\
320 & QPSK & $1 / 2$ & No & 1 \\
400 & QPSK & $5 / 8$ & No & 1 \\
480 & QPSK & $3 / 4$ & No & 1 \\
\hline
\end{tabular}

occupies a bandwidth of at least $500 \mathrm{MHz}$ in compliance with the FCC regulations. The UWB system employs OFDM with $N$ subcarriers, which are modulated using quadrature phase shift keying (QPSK). At each OFDM symbol period, the modulated symbol is transmitted over one of the $S$ subbands. These symbols are time-interleaved across subbands. Different data rates are achieved by using different channel coding, frequency spreading, or time spreading rates [7]. The frequency domain spreading is obtained by choosing conjugate symmetric inputs to the IFFT. Specifically, $N / 2$ complex symbols are transmitted in the first half of the subcarriers, and their conjugate symmetric symbols are transmitted in the second half of the subcarriers. The time domain spreading is achieved by transmitting one OFDM symbol followed by a permutation of that OFDM symbol, i.e., transmitting the same information twice during two OFDM symbol periods. The receiver combines the information transmitted via different times or frequencies to increase the signal-to-noise ratio (SNR) of received data.

As listed in Table I, the multiband UWB system provides data rates ranging from 53.3 Mbps to $480 \mathrm{Mbps}$. For the rates not higher than $80 \mathrm{Mbps}$, both time and frequency spreadings are performed, yielding the overall spreading gain of four. For the rates between 106.7 and $200 \mathrm{Mbps}$, only time-domain spreading is utilized which results in the overall spreading gain of two. The system with data rates higher than $200 \mathrm{Mbps}$ exploits neither frequency nor time spreading, and the overall spreading gain is one. Forward error correction codes with coding rates of $1 / 3,11 / 32,1 / 2,5 / 8$ or $3 / 4$ are employed to 
provide different channel protections with various data rates.

In what follows, we will describe the channel model and system model for the multiuser multiband UWB system.

\section{A. Channel Model}

The channel model specified in the IEEE 802.15.3a standard [14] is a modified version of the Saleh-Valenzuela (SV) model for indoor channels [15]. The channel impulse response for the $k^{t h}$ user at time $t$ can be represented by

$$
h_{k}(t)=X_{k} \sum_{m=0}^{M_{k}} \sum_{l=0}^{L_{k}} \alpha_{k}(m, l) \delta\left(t-T_{k}(m)-\tau_{k}(m, l)\right),
$$

where $X_{k}$ is the log-normal shadowing, and $\alpha_{k}(m, l)$ denotes the gain of the $l^{t h}$ multipath component in the $m^{t h}$ cluster. The time duration $T_{k}(m)$ represents the delay of the $m^{t h}$ cluster, and $\tau_{k}(m, l)$ is the delay of the $l^{t h}$ path in the $m^{t h}$ cluster relative to the cluster arrival time. The cluster arrivals and the path arrivals within each cluster can be modeled as Poisson distribution with rate $\Lambda_{k}$ and rate $\lambda_{k}\left(\lambda_{k}>\Lambda_{k}\right)$, respectively. In (1), $M_{k}$ is the number of clusters, and $L_{k}$ is the number of paths within each cluster. The path amplitude $\left|\alpha_{k}(m, l)\right|$ follows the log-normal distribution, whereas the phase $\angle \alpha_{k}(m, l)$ is a uniform random variable over $[0,2 \pi)$. The model parameters corresponding to several scenarios are provided in [14]. With the choice of cyclic prefix length greater than the duration of the channel impulse response, OFDM allows for each UWB subband to be divided into a set of $N$ orthogonal narrowband channels. The baseband frequency response at the $n^{t h}(n=0,1, \ldots, N-1)$ subcarrier is

$$
H_{k}(n)=X_{k} \sum_{m=0}^{M_{k}} \sum_{l=0}^{L_{k}} \alpha_{k}(m, l) e^{-\mathrm{j} 2 \pi n \Delta f\left(T_{k}(m)+\tau_{k}(m, l)\right)},
$$

where $\mathrm{j}=\sqrt{-1}$, and $\Delta f$ is the frequency separation between two adjacent subcarriers. It is worth noting that for most WPAN applications, the transmitter and receiver are stationary [16]. As a result, UWB channel is very slowly fading. The standard channel model assumes that the channel stays either completely static, or is time-invariant during the transmission of each packet [14], [16]. We assume that the channel state information is known at both the transmitter and the receiver.

\section{B. System Model: Subband Assignment and Power Allocation}

We consider a multiuser multiband UWB scenario where $K$ users simultaneously transmit their information. The $k^{\text {th }}$ user has the data rate $R_{k}$, which can be any value specified in Table I. As shown in Table I, if the rate is higher than $200 \mathrm{Mbps}$, there is no time spreading; otherwise, the timedomain spreading operation is performed with a spreading factor of two. In this case, any time-frequency code with a period of two can guarantee that each user will achieve the additional diversity by transmitting the same information over two OFDM blocks. The time-frequency codes with period longer than two can also be used to improve the multiple access capability for asynchronous UWB wireless networks [7]. To simplify the problem formulation, we consider in this paper a multiband UWB system employing time-frequency codes of length two. The extension to UWB systems with longer time-frequency codes is straightforward.

In order to specify in which subbands each user can transmit its information, we define a $K \times S$ assignment matrix $\mathbf{A}$, whose $(k, s)^{t h}$ element is denoted by $a_{k s}$, for $k=1,2, \ldots, K$ and $s=1,2, \ldots, S$. This $a_{k s}$ represents the number of OFDM symbols that user $k$ is allowed to transmit on the $s^{t h}$ subband during two OFDM symbol periods. Assuming that each user utilizes one subband per transmission, $a_{k s}$ can take any value from the set $\{0,1,2\}$. However, when the $k^{t h}$ the data rate of the user is less than or equal to $200 \mathrm{Mbps}$, we need to ensure that the band hopping is performed to obtain the diversity from time spreading. In this case, $a_{k s}$ is restricted to $a_{k s} \in\{0,1\}$. Thus, the element of assignment matrix satisfies [17]

$$
a_{k s} \in \phi\left(R_{k}\right)= \begin{cases}\{0,1\}, & R_{k} \leq 200 \mathrm{Mbps} ; \\ \{0,1,2\}, & R_{k}>200 \mathrm{Mbps} .\end{cases}
$$

During each OFDM symbol period, one user will occupy one subband. Since we consider the duration of two OFDM blocks, the assignment strategy needs to satisfy

$$
\sum_{s=1}^{S} a_{k s}=2, \quad k=1,2, \ldots, K .
$$

In addition, to minimize the multiple access interference, each subband is assigned to a specific user at a time, and hence each subband can be used at most twice during two OFDM symbol periods. Therefore, the subband assignment also follows

$$
\sum_{k=1}^{K} a_{k s} \leq 2, \quad s=1,2, \ldots, S
$$

Let $P_{k}^{s}(n)$ denote the transmit power of the $k^{t h}$ user at subcarrier $n$ of the $s^{t h}$ subband. Accordingly, the SNR of user $k$ at the $s^{t h}$ subband and the $n^{t h}$ subcarrier is given by

$$
\Gamma_{k}^{s}(n)=\frac{P_{k}^{s}(n) G_{k}^{s}(n)}{\sigma_{k}^{2}},
$$

where $G_{k}^{s}(n)$ is the corresponding channel gain. We can express $G_{k}^{s}(n)$ as

$$
G_{k}^{s}(n)=\left|H_{k}^{s}(n)\right|^{2}\left(\frac{4 \pi d_{k}}{\lambda_{k}^{s}}\right)^{-\nu},
$$

in which $H_{k}^{s}(n)$ is the channel frequency response at subband $s$ and subcarrier $n, \nu$ is the propagation loss factor, $d_{k}$ represents the distance between the transmitter and receiver, $\lambda_{k}^{s}=3 \times 10^{8} / f_{c, k}^{s}$ is the wavelength of the transmitted signal, and $f_{c, k}^{s}$ is the center frequency of the waveform. In (6), $\sigma_{k}^{2}$ denotes the noise power at each subcarrier, defined as

$$
\sigma_{k}^{2}=2 \times 10^{\left(-174+10 \log _{10}\left(R_{k}\right)+N_{F}\right) / 10},
$$

where $R_{k}$ is the data rate of the $k^{t h}$ user, and $N_{F}$ is the received noise figure referred to the antenna terminal [7]. As in the multiband standard, we assume that the noise power $\sigma_{k}^{2}$ is the same for every subcarrier within each subband. We assume an ideal band hopping such that the signal transmitted over different subband undergo independent fading, and there is no multiple access interference.

Due to the consideration for the simple transceiver of UWB, 
the current standard assumes that there is no bit loading and the power is equally distributed across subcarriers within each subband. Similarly, we assume that $P_{k}^{s}(n)=P_{k}^{s}\left(n^{\prime}\right)$ for any $0 \leq n, n^{\prime} \leq N-1$. Denote

$$
P_{k}^{s}(n)=P_{k}^{s}, \quad n=0,1, \ldots, N-1,
$$

then the $K \times S$ power allocation matrix can be defined as $[\mathbf{P}]_{k s}=P_{k}^{s}$, in which $(k, s)^{t h}$ component represents the transmit power of the $k^{\text {th }}$ user in subband $s$.

\section{Proposed Multiband}

\section{Channel Allocation Algorithm}

In the multiband frequency band plan [7], the subband center frequencies span a wide range from $3.43 \mathrm{GHz}$ to $10.3 \mathrm{GHz}$. Consequently, different subbands tend to undergo different fading and propagation loss. Additionally, the channel condition for a specific subband may be good for more than one user. Therefore, to efficiently reduce the power consumption, we need to optimize the subband assignment matrix $\mathbf{A}$ and power allocation matrix $\mathbf{P}$ under some practical constraints.

In this section, first, we derive a generalized SNR expression for various UWB transmission modes. Second, we provide a necessary condition for the SNR so as to satisfy the PER requirement. Then, we propose a problem formulation to minimize the overall transmit power provided that all users achieve their requested data rates and desired PER, while the transmit power level is below the FCC limitation and rate parameters are according to the standard proposal given in Table I. We develop a fast suboptimal scheme to solve the proposed problem. Finally, to ensure the system feasibility, we develop a joint rate adaptation, subband assignment, and power allocation algorithm.

\section{A. Generalized SNR for Different Transmission Modes}

Assuming that the channel state information is perfectly known at the receiver, the receiver employs a maximum ratio combiner (MRC) to combine the information transmitted via different times or frequencies. As a result, the average SNR at the output of MRC depends not only on the channel coding rate, but also the time and frequency spreading factors. The following proposition provides a generalized expression of the average SNR for any data rates.

Proposition 1 Assume maximum ratio combining and $P_{k}^{s}(n)=P_{k}^{s}$ for all subcarrier $n$, then the average SNR of the $k^{\text {th }}$ user is given by

$$
\bar{\Gamma}_{k}=\sum_{s=1}^{S} a_{k s} P_{k}^{s} F_{k}^{s}
$$

where

$$
F_{k}^{s} \triangleq \frac{b_{k}}{N \sigma_{k}^{2}} \sum_{n=0}^{N-1} G_{k}^{s}(n),
$$

and $b_{k}$ is a constant that depends on the data rate of the $k^{t h}$ user as follows:

$$
b_{k}= \begin{cases}2, & R_{k} \leq 80 \text { Mbps } \\ 1, & 80<R_{k} \leq 200 \text { Mbps } \\ 1 / 2, & R_{k}>200 \text { Mbps }\end{cases}
$$

Proof: Recall that when $R_{k}$ is not higher than $80 \mathrm{Mbps}$, the information is spread across both time and frequency with the overall spreading gain of four. Consequently, the total SNR for the $k^{t h}$ user at subcarrier $n, n=0,1, \ldots, N / 2-1$, is

$$
\Gamma_{k}(n)=\sum_{s=1}^{S} a_{k s}\left[\Gamma_{k}^{s}(n)+\Gamma_{k}^{s}(n+N / 2)\right] .
$$

Note that the SNR in (13) is based on the assumptions of no multiuser interference and no correlation among the data bits; it leads to an upper bound on the performance. Average (13) over $N / 2$ subcarriers, resulting in the average SNR

$$
\bar{\Gamma}_{k}=\frac{1}{N / 2} \sum_{n=0}^{N / 2-1} \Gamma_{k}(n)=\frac{1}{N / 2} \sum_{n=0}^{N-1} \sum_{s=1}^{S} a_{k s} \Gamma_{k}^{s}(n) .
$$

By substituting (6) into (14) and assuming $P_{k}^{s}(n)=P_{k}^{s}$, we obtain

$$
\begin{aligned}
\bar{\Gamma}_{k} & =\frac{2}{N} \sum_{n=0}^{N-1} \sum_{s=1}^{S} a_{k s} P_{k}^{s} \frac{G_{k}^{s}(n)}{\sigma_{k}^{2}} \\
& =\sum_{s=1}^{S} a_{k s} P_{k}^{s}\left(\frac{2}{N \sigma_{k}^{2}} \sum_{n=0}^{N-1} G_{k}^{s}(n)\right) .
\end{aligned}
$$

When $R_{k}$ is between 106.7 and $200 \mathrm{Mbps}$, only time spreading is performed, and hence the total SNR at subcarrier $n, n=0,1, \ldots, N-1$, becomes

$$
\Gamma_{k}(n)=\sum_{s=1}^{S} a_{k s} \Gamma_{k}^{s}(n)=\sum_{s=1}^{S} a_{k s} \frac{P_{k}^{s}(n) G_{k}^{s}(n)}{\sigma_{k}^{2}} .
$$

Thus, the average SNR can be obtained from (16) as

$$
\bar{\Gamma}_{k}=\frac{1}{N} \sum_{n=0}^{N-1} \Gamma_{k}(n)=\sum_{s=1}^{S} a_{k s} P_{k}^{s}\left(\frac{1}{N \sigma_{k}^{2}} \sum_{n=0}^{N-1} G_{k}^{s}(n)\right) .
$$

For $R_{k}$ higher than $200 \mathrm{Mbps}$, there is no spreading and the average SNR of the $k^{t h}$ user is simply the average of $\Gamma_{k}^{s}(n)$ over $N$ subcarriers and two subbands, i.e.,

$$
\begin{aligned}
\bar{\Gamma}_{k} & =\frac{1}{2 N} \sum_{n=0}^{N-1} \sum_{s=1}^{S} a_{k s} \Gamma_{k}^{s}(n) \\
& =\sum_{s=1}^{S} a_{k s} P_{k}^{s}\left(\frac{1}{2 N \sigma_{k}^{2}} \sum_{n=0}^{N-1} G_{k}^{s}(n)\right) .
\end{aligned}
$$

Express (15), (17) and (18) in terms of $F_{k}^{s}$ defined in (11) leading to the results in (10).

\section{B. PER and Rate Constraint}

A common performance requirement of UWB systems is to offer packet transmission with an error probability less than a desired threshold value. The PER metric is directly related to the bit error rate (BER) performance, which in turn depends on the SNR at the output of the MRC. By keeping the SNR level higher than a specific value, the PER can be ensured to be lower than the PER threshold. In the sequel, we provide a necessary condition for the average SNR so as to satisfy the PER requirement.

Suppose the maximum PER is $\varepsilon$ and the packet length is $L$ bits, then the bit error probability after the channel decoder 
for the $k^{t h}$ user, $\mathcal{P}_{k}$, needs to satisfy

$$
1-\left(1-\mathcal{P}_{k}\right)^{L} \leq \varepsilon
$$

By the assumptions of the use of convolutional coding and Viterbi decoding with perfect interleaving, $\mathcal{P}_{k}$ is given by [18]

$$
\mathcal{P}_{k} \leq \sum_{d=d_{\text {free }}}^{\infty} a_{d} \mathcal{P}_{k}(d)
$$

where $d_{\text {free }}$ is the free distance of the convolutional code, $a_{d}$ denotes the total number of error events of weight $d$, and $\mathcal{P}_{k}(d)$ represents the probability of choosing the incorrect path with distance $d$ from the correct path. With hard-decision decoding, $\mathcal{P}_{k}(d)$ is related to the average BER, $\bar{B}_{k}$, as [18]

$$
\mathcal{P}_{k}(d)=\sum_{l=(d+1) / 2}^{d}\left(\begin{array}{l}
d \\
l
\end{array}\right) \bar{B}_{k}^{l}\left(1-\bar{B}_{k}\right)^{d-l}
$$

when $d$ is odd, and

$$
\mathcal{P}_{k}(d)=\sum_{l=\frac{d}{2}+1}^{d}\left(\begin{array}{l}
d \\
l
\end{array}\right) \bar{B}_{k}^{l}\left(1-\bar{B}_{k}\right)^{d-l}+\frac{1}{2}\left(\begin{array}{l}
d \\
\frac{d}{2}
\end{array}\right) \bar{B}_{k}^{\frac{d}{2}}\left(1-\bar{B}_{k}\right)^{\frac{d}{2}}
$$

when $d$ is even.

The average BER $\bar{B}_{k}$ can be obtained by averaging the conditional BER over the probability density function of the SNR at the output of MRC. With $\Gamma_{k}$ denoting the instantaneous SNR at the MRC output, the conditional BER is [18]

$$
B_{k}\left(\Gamma_{k}\right)=\mathrm{Q}\left(\sqrt{\Gamma_{k}}\right)
$$

where $\mathrm{Q}(\cdot)$ is the Gaussian error function. From (19) and (20), we can see that for a given value of PER threshold $\varepsilon$, a corresponding BER threshold can be obtained. Since the error probability $\mathcal{P}_{k}$ in (20) is related to the coding rate through the parameters $d_{\text {free }}$ and $a_{d}$, the BER requirement depends not only on the value of $\varepsilon$, but also on the data rate $R_{k}$. This implies that the SNR threshold is also a function of both $\varepsilon$ and $R_{k}$. Let $\gamma\left(\varepsilon, R_{k}\right)$ be the minimum SNR of the $k^{t h}$ user that is required to achieve the data rate $R_{k}$ with PER less than $\varepsilon$. Then, the necessary condition for the average SNR (defined in (10)) to satisfy the PER requirement is given by

$$
\bar{\Gamma}_{k}=\sum_{s=1}^{S} a_{k s} P_{k}^{s} F_{k}^{s} \geq \gamma\left(\varepsilon, R_{k}\right)
$$

\section{Problem Formulation}

The optimization goal is to minimize the overall transmit power subject to the PER, rate, and FCC regulation constraints. Recall from (3) that the assignment matrix $\mathbf{A}$ has $a_{k s} \in \phi\left(R_{k}\right), \forall k, s$. We can formulate the problem as follows:

$$
\min _{\mathbf{A}, \mathbf{P}} P_{\text {sum }}=\sum_{k=1}^{K} \sum_{s=1}^{S} a_{k s} P_{k}^{s}
$$

s.t. $\left\{\begin{array}{l}\text { Rate and PER: } \sum_{s=1}^{S} a_{k s} P_{k}^{s} F_{k}^{s} \geq \gamma\left(\varepsilon, R_{k}\right), \forall k ; \\ \text { Assignment (4): } \sum_{s=1}^{S} a_{k s}=2, \forall k \\ \text { Assignment (5): } \sum_{k=1}^{K} a_{k s} \leq 2, \forall s \\ \text { Power: } P_{k}^{s} \leq P_{\max }, \forall k, s,\end{array}\right.$ where the first constraint in (23) is to ensure rate and PER requirements. The second and third constraints are described in Section II-B. The last constraint is related to the limitation on transmit power spectral density of $-41.3 \mathrm{dBm} / \mathrm{MHz}$, according to FCC Part 15 rules [1]. Here, $P_{\max }$ is the maximum transmit power taking into consideration the effects such as peak-to-average power ratio (PAPR), i.e., $P_{\max }$ is the maximum power considering both the average maximum power allowed by the FCC and the PAPR of OFDM signals.

If the elements in the assignment matrix $\mathbf{A}$ are binary, the problem defined in (23) can be viewed as a generalized form of generalized assignment problem [19] which is $N P$ hard. Since the components of $\mathbf{A}$ can be 0,1 , or 2 , the problem is an even harder integer programming problem. So the existing channel assignment approaches, e.g. in [20], are not applicable in (23). Although the optimal solution can be found through full search, it is computationally expensive. To overcome the complexity issue, we propose in the subsequent subsection a fast suboptimal scheme, which is near optimal but has very low computational complexity.

\section{Subband Assignment and Power Allocation Algorithm}

The basic idea is a greedy approach to assign $a_{k s}$ for a user step by step, so that the power consumption is minimized. The initialization is to set $\mathbf{A}=\mathbf{0}_{K \times S}$, define the user optimization list $K_{\text {live }}=\{1,2, \ldots, K\}$, and define the subband optimization list $S_{\text {live }}=\{1,2, \ldots, S\}$. First, each user makes a hypothesis that it can assign its transmission into different subbands regarding absence of other users. For each hypothesis, a dummy overall transmission power $P_{d u m m y}^{k}$ is calculated by finding the minimum power among all possible subbands such that the BER performance requirement of user $k$ is satisfied. The user with the highest dummy overall transmit power to achieve its rate will be assigned first, so that the best channel is assigned to the user that can reduce the overall power most. Then, this user is removed from the optimization list $K_{\text {live }}$. Since each subband can only accommodate one user per symbol period and we consider two OFDM symbol periods, when a subband is assigned twice, this subband is removed from the optimization list $S_{\text {live }}$. Then, we go to the first step for the rest of the users to assign their transmissions into the rest of the subbands. This iteration is continued until all users are assigned with their subbands, i.e., $K_{\text {live }}=\emptyset$. Finally, the algorithm checks if the maximum power is larger than the power limitation. If yes, an outage is reported; otherwise, the final values of $\mathbf{A}$ and $\mathbf{P}$ are obtained. The proposed algorithm can be described as follows:

Initialization: $a_{k s}=0, \forall k, s, K_{\text {live }}=\{1, \ldots, K\}, S_{\text {live }}=$ $\{1, \ldots, S\}$

Iteration: Repeat until $K_{\text {live }}=\emptyset$ or $S_{\text {live }}=\emptyset$

1) For $k \in K_{\text {live }}$

$$
P_{\text {dummy }}^{k}=\min \sum_{s=1}^{S} a_{k s} P_{k}^{s} \quad \text { s.t. } a_{k s} \in S_{\text {live }}
$$

End

2) Select $k^{\prime}$ with the maximal $P_{d u m m y}^{k}, \forall k$, assign the corresponding $a_{k^{\prime} s}$ to $\mathbf{A}$, and update $\mathbf{P}$.

3) $K_{\text {live }}=K_{\text {live }} \backslash k^{\prime}$

4) If $\sum_{k=1}^{K} a_{k s^{\prime}}=2, S_{\text {live }}=S_{\text {live }} \backslash s^{\prime}, \forall s^{\prime}$. 
End: If $\left(\max (\mathbf{P})>P_{\text {max }}\right)$ or $\left(S_{\text {live }}=\emptyset\right.$ and $\left.K_{\text {live }} \neq \emptyset\right)$, an outage is reported. Otherwise, return $\mathbf{A}$ and $\mathbf{P}$.

The complexity of the proposed algorithm is only $O\left(K^{2} S\right)$. Although the algorithm is suboptimal, simulation results illustrated in the succeeding section shows that the proposed fast suboptimal algorithm has very close performances to the optimal solutions obtained by full search. Another complexity issue is that for the proposed scheme, power control is needed for each subband ${ }^{1}$. This will increase the system complexity slightly, but from the simulation results, we can see that the performance improvement is significant. Moreover, the proposed algorithm can be implemented by the master node to manage the power and subband usages of all users in a UWB picocell system, as adopted in the IEEE 802.15.3a standard [8]. The signaling information needed to be broadcasted at the master node includes the band hopping sequence of each user and the corresponding transmit power. The algorithm is updated when a new user joins the network or when the channel link quality of each user changes considerably. In each update, the algorithm requires the channel state information for all considered subbands (instead of three subbands as in the standard multiband scheme) between every transmitters and the receiver. Such update does not frequently occurs thanks to the small size of the picocell and the stationary nature of most transceivers in WPAN applications.

\section{E. Joint Rate Assignment and Resource Allocation Algorithm}

Since the transmit power in each subband is limited by maximal power $P_{\max }$, solutions to (23) may not exist in some situations, such as when the requested rates of the users are high but the channel conditions are poor. Under such conditions, some desired rates of the users cannot be satisfied, and we call that the system is infeasible. When the system is not feasible, the requested rates need to be reduced. Here, we develop a joint rate assignment and power controlled channel allocation algorithm that is able to obtain the feasible solutions adaptively when the initial system is not feasible for the rate requirements of the users. Basically, the proposed algorithm comprises two main stages, namely resource allocation and rate adaptation stages. Fig. 2 shows the flow chart diagram of the proposed algorithm.

At the initialization step, the data rate of the $k^{\text {th }}$ user, $R_{k}, k=1,2, \ldots, K$ is set to its requested rate. After the initial setting, the first stage is to perform the subband and power allocation using the algorithm described in the previous subsection. If there is a solution for this assignment, then it is done. Otherwise, an outage will be reported, indicating the requested rates of the users are too high for the current channel conditions. In this case, we proceed to the second stage where the rate adaptation is performed.

In the rate adaptation stage, the algorithm chooses only one user, reduces its rate to the next lower rate as listed in Table I. In order to specify which user to be selected we consider three different goals, namely maximizing overall rate,

\footnotetext{
${ }^{1}$ But no power control or bit loading for subcarriers within each subband
}

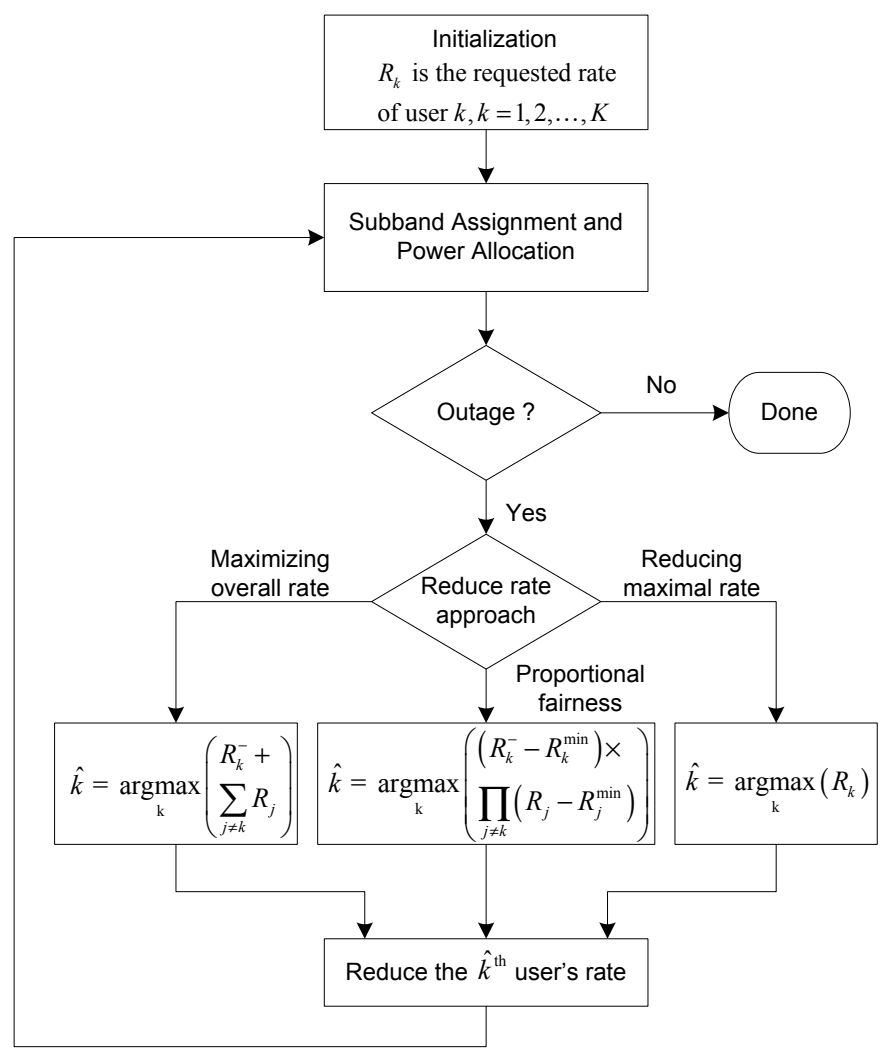

Fig. 2. Flow chart of the proposed algorithm.

achieving proportional fairness $[21]^{2}$, and reducing maximal rate. In particular, given the data rate of the $k^{t h}$ user, $R_{k}$, we denote its one-step reduced rate by $R_{k}^{-}$. For instance, from Table I, the reduced rate $R_{k}^{-}$corresponding to a rate $R_{k}=320 \mathrm{Mbps}$ is $R_{k}^{-}=200 \mathrm{Mbps}$. Note that when the rate $R_{k}$ reaches the minimum allowable rate of $53.3 \mathrm{Mbps}$, we let $R_{k}^{-}=R_{k}$, i.e., the rate $R_{k}$ is not further reduced. Then, the user $\hat{k}$ whose rate will be reduced can be determined according to the performance goals as:

- Maximizing overall rate: $\arg \max _{k} R_{k}^{-}+\sum_{j=1, j \neq k}^{K} R_{j}$.

- Proportional fairness: $\arg \max _{k} \prod_{j=1, j \neq k}^{K}\left(R_{j}-R_{j}^{\min }\right) \times$ $\left(R_{k}^{-}-R_{k}^{\min }\right)$.

- Reducing maximal rate: $\hat{k}=\arg \max _{k}\left(R_{k}\right)$.

where $R_{k}^{\min }$ denotes a minimal rate requirement for user $k$. With maximizing overall rate approach, the overall system rate is maximized in every reduction step. In case of the proportional fairness approach, the product of rates minus minimal rate requirements [21] is maximized. For reducing maximal rate approach, the highest rate in the system will be reduced. Note that if there is still no solution to the assignment after the rates of all users are reduced to the minimum allowable rate, then an outage is reported. This indicates that the system under the current channel conditions cannot support the transmission of all $K$ users at the same time. The proposed joint resource allocation and rate adaptation

\footnotetext{
${ }^{2}$ Note that proportional fairness is achievable when the utility is a log function. In this paper, we have discrete and non-convex case, so the same product form is used as the system performance goal instead of the log function. From the simulations, this goal achieves tradeoff of performances and fairness between the maximal rate goal and reducing maximal rate goal.
} 
algorithm is summarized as follows.

Initialization: Iteration index $n^{\prime}=0, R_{k}(0)=$ requested rate of user $k, k=1,2, \ldots K$

Iteration:

1) Given $R_{k}\left(n^{\prime}\right)$, solve subband assignment and power allocation problem in (23).

2) If (23) has a solution, the algorithm ends. Otherwise,

- If $R_{k}\left(n^{\prime}\right)=R_{k}^{-}\left(n^{\prime}\right), \forall k$, then an outage is reported and the algorithm ends.

- Determine $\hat{k}$.

- Update the rates:

$$
R_{k}\left(n^{\prime}+1\right)= \begin{cases}R_{k}^{-}\left(n^{\prime}\right), & k=\hat{k} \\ R_{k}\left(n^{\prime}\right), & \text { otherwise. }\end{cases}
$$

- Set $n^{\prime}=n^{\prime}+1$.

\section{Simulation Results}

To illustrate the performance of the proposed schemes, we perform simulations for multiband UWB systems with $N=$ 128 subcarriers, $S=14$ subbands, and the subband bandwidth of $528 \mathrm{MHz}$. Following the IEEE 802.15.3a standard proposal [7], we utilize the subbands with center frequencies $2904+$ $528 \times n_{b} \mathrm{MHz}, n_{b}=1,2, \ldots, 14$. The OFDM symbol is of duration $T_{F F T}=242.42 n \mathrm{~s}$. After adding the cyclic prefix of length $T_{C P}=60.61 \mathrm{~ns}$ and the guard interval of length $T_{G I}=$ $9.47 n \mathrm{~s}$, the symbol duration becomes $T_{S Y M}=312.5 \mathrm{~ns}$. As in [7], the convolutional encoder with a constraint length of 7 is used to generate different channel coding rates. The maximum transmit power is $-41.3 \mathrm{dBm} / \mathrm{MHz}$, and the PER is maintained such that PER $<8 \%$ for a 1024 byte packet. The average noise power follows (8) with $N_{F}=6.6 \mathrm{~dB}$, and the propagation loss factor is $\nu=2$.

We consider a multiuser scenario in which each user is located at a distance of less than 4 meters from the central base station. The performance is evaluated in multipath channel environments specified in the IEEE 802.15.3a channel modeling sub-committee report [14]. We employ channel model 1 and 2 , which are based on channel measurements over the range of 0-4 meters. The simulated channels were constant during the transmission of each packet, and independent from one packet to another. In each simulation, we averaged over a minimum of 50000 channel realizations.

\section{A. Subband Assignment and Power Allocation}

In this subsection, we present the average transmit power and the outage probability curves for multiband UWB systems. Here, the outage probability is the probability that the requested rate cannot be supported under the constraints in (23). We compare the performances of the proposed scheme with those of the current multiband scheme in the standards proposal [7].

For Fig. 3(a) and Fig. 3(b), the number of users is fixed to $K=3$, while each user is randomly located at the distance of 1 to 4 meters from the base station. In Fig. 3(a), we illustrate the average transmit power as a function of the data rates for standard multiband scheme, the proposed fast suboptimal scheme, and the optimal scheme obtained by full search. It is apparent that the proposed algorithm can achieve almost the same performance to the optimal scheme. In addition, the

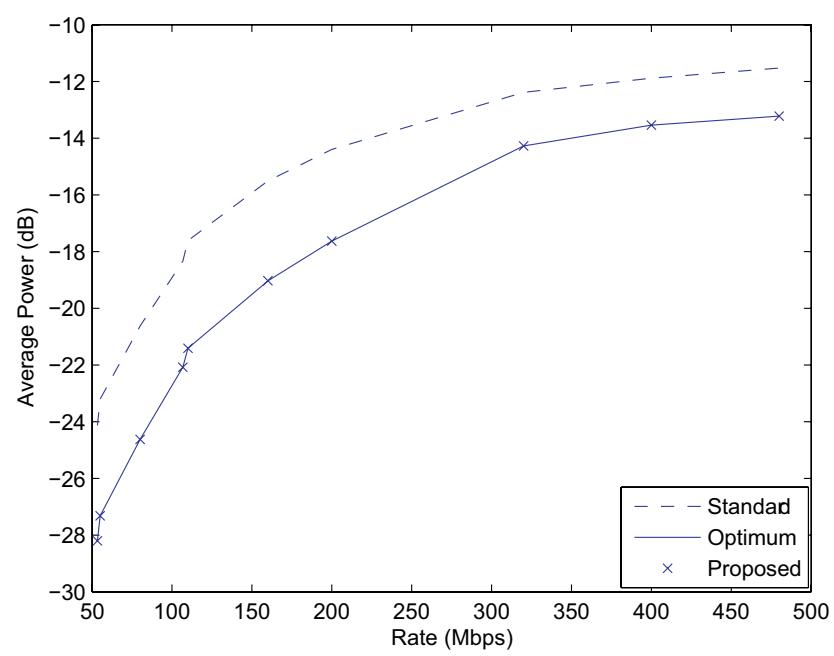

(a) Average power vs. rates

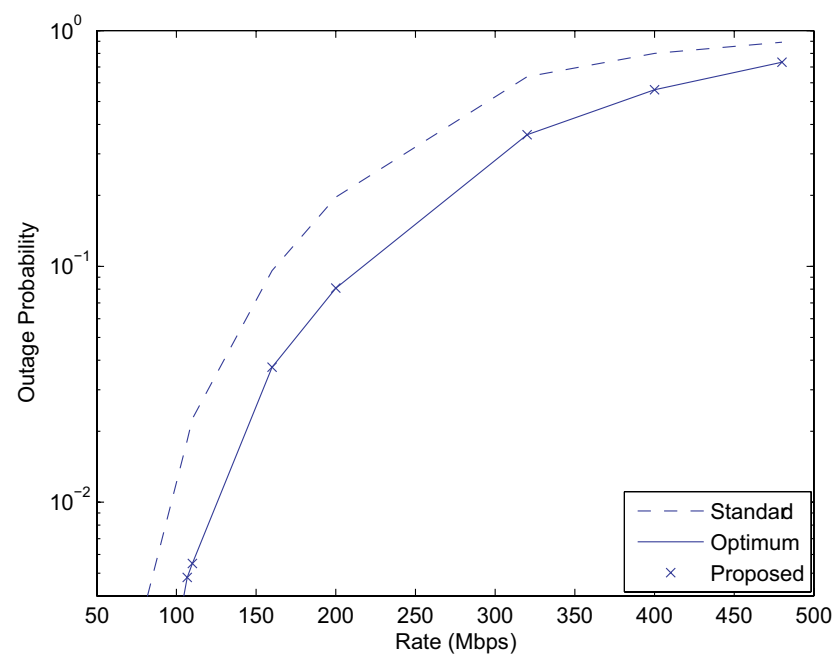

(b) Outage probability vs. rates

Fig. 3. Performances of three-user system with random location.

proposed algorithm greatly reduces the average transmit power compared to that in the standard proposal. The performance gain in term of power reduction that is achieved by the proposed scheme, compared with the standard scheme, can be computed as $\left(P_{\text {standard }}-P_{\text {proposed }}\right) / P_{\text {standard }}$, where $P_{\text {standard }}$ and $P_{\text {proposed }}$ denote the average powers for the standard and the proposed schemes, respectively. The results in Fig. 3(a) show that both fast suboptimal and optimal approach can reduce about $60 \%$ of average transmit power at low rates (53.3-200 Mbps) and up to 35\% at high rates (320-480 Mbps). Notice that the curves are not smooth because of the discrete nature of the problem. Fig. 3(b) shows the outage probability versus the transmission rates. We can see that the proposed scheme achieves lower outage probability than that of the standard multiband scheme for any rates. For instance, at $110 \mathrm{Mbps}$, the outage probability of the proposed scheme is $5.5 \times 10^{-3}$, whereas that of the standard multiband scheme is $2.3 \times 10^{-2}$.

We also consider a multiuser system with different number of users, each located at a fixed position of about 4 meters from the base station. Specifically, the distance between the 


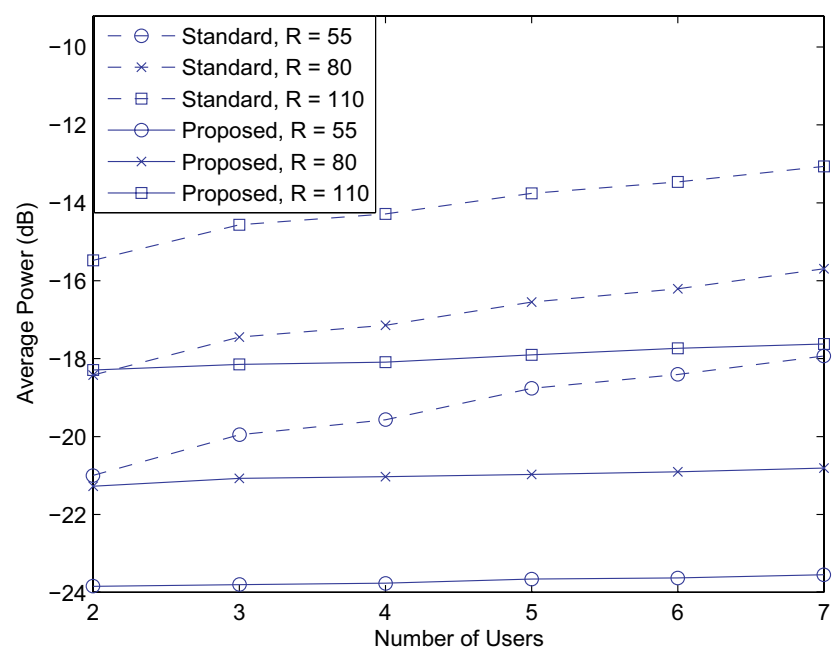

(a) Average power vs. number of users

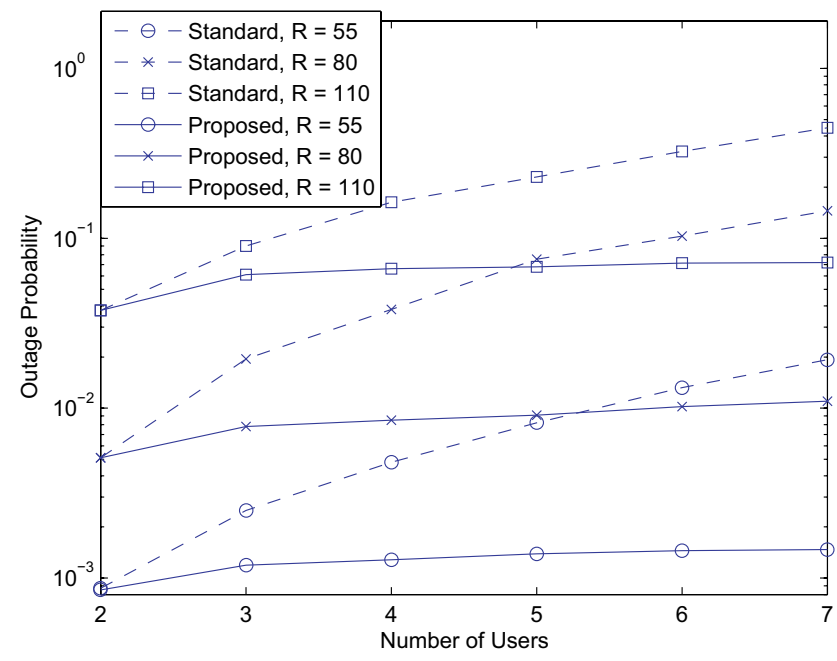

(b) Outage probability vs. number of users

Fig. 4. Performances of multiple-user system.

$k^{t h}$ user and the base station is specified as $d_{k}=4-0.1(k-1)$ for $k=1,2, \ldots, K$. In Fig. 4(a) and Fig. 4(b), we show the average transmit power and outage probability as functions of number of users for the data rates of 55, 80, and $110 \mathrm{Mbps}$. In both figures, we use the standard multiband scheme and the proposed scheme. We can observe from Fig. 4(a) that the transmit power increases with the number of users. This results from the limited available subbands with good channel conditions. When the number of users is large, some users have to occupy the subbands with worse channel conditions. Comparing the proposed algorithm with standard multiband approach, we can see that the proposed scheme achieves lower transmit power for all the rate requirements.

Fig. 4(b) shows that the outage probability increases with the number of users. This is due to the fact that as the number of users increases, the system is more crowded and may not be feasible to support all these users at all times. Observe that at any rate, the performance of the standard multiband scheme degrades as the number of users increases. On the other hand, when the proposed scheme is employed, the effect of the number of users to the outage probability is insignificant

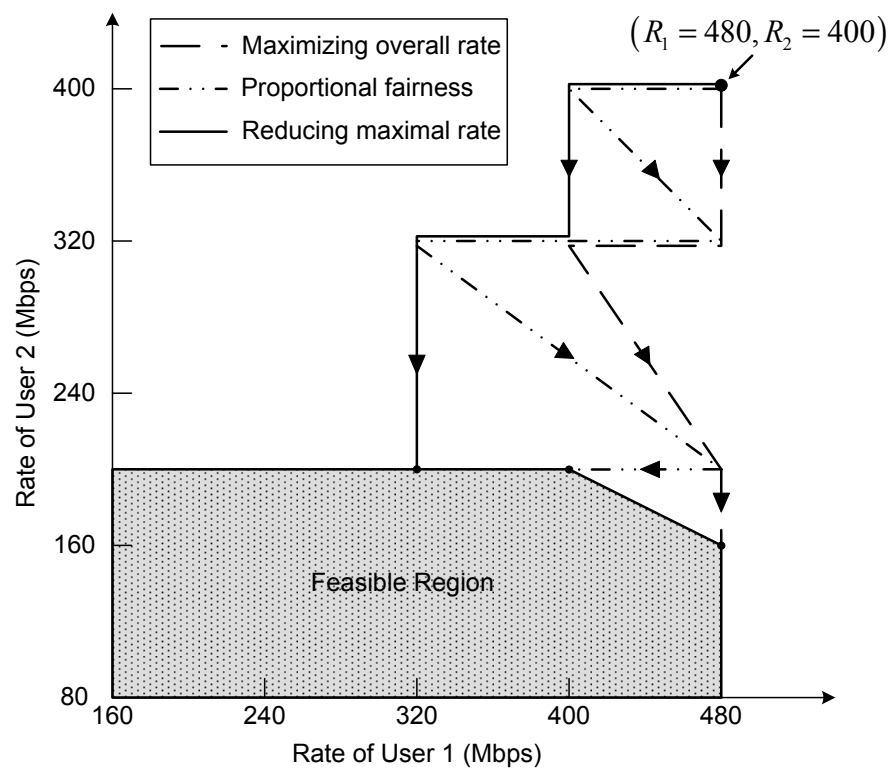

Fig. 5. One realization of rate adaptation for two-user system.

when the rates are not higher than $110 \mathrm{Mbps}$. As we can see, the proposed algorithm achieves smaller outage probabilities than those of the standard scheme under all conditions.

\section{B. Joint Rate Assignment and Resource Allocation}

This subsection illustrates the performances of the proposed joint rate assignment and resource allocation algorithm for multiband system. We consider a multiuser system with different number of users. Each user is randomly located at the distance of 1 to 4 meters from the base station. The requested rates of users are also randomly selected from the set $\{200,320,400,480\} \mathrm{Mbps}$, and the minimum rate requirement is $R_{k}^{\min }=50 \mathrm{Mbps} \forall k$ for proportional fairness goal. The joint rate assignment and resource allocation algorithm proposed in Section III-E is performed for each set of requested rates and channel conditions.

Fig. 5 illustrates one realization of rate adaptation for a two-user system with three different goals. The shaded area represents the feasible range for $R_{1}$ and $R_{2}$ in the current channel conditions. In this example, the requested rates are $R_{1}=480$ and $R_{2}=400 \mathrm{Mbps}$, and both users are located at about 4 meters from the base station. We can observe from Fig. 5 that the reducing maximal rate approach has lowest overall rate in every adaptation step. This is because the highest rate in the system can always be reduced. On the other hand, the maximizing overall rate approach tends to reduce the lower rate since most low rates have smaller decreasing step size than high rates. Although the maximizing overall rate approach always yields superior system performance, it is unfair to those applications with low data rates. The proportional fairness goal provides the performance that is between the maximizing overall rate approach and reducing maximal rate approach.

Fig. 6(a) and Fig. 6(b) show the average system performance versus the number of users. In Fig. 6(a), we present the performances in term of the average data rates of the users. We can see that the average rates of all three approaches decrease when the number of users increases. This is due to the 


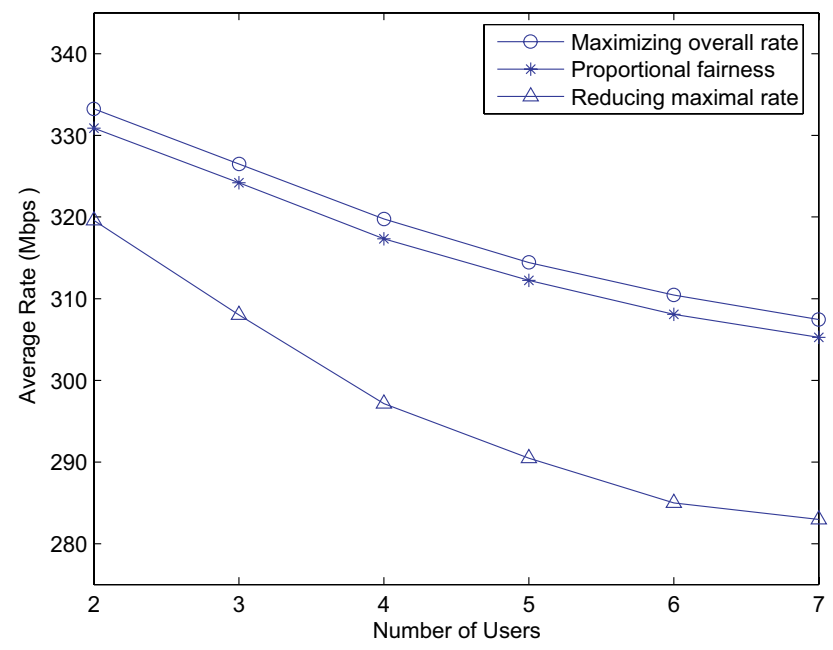

(a) Average rate vs. number of users

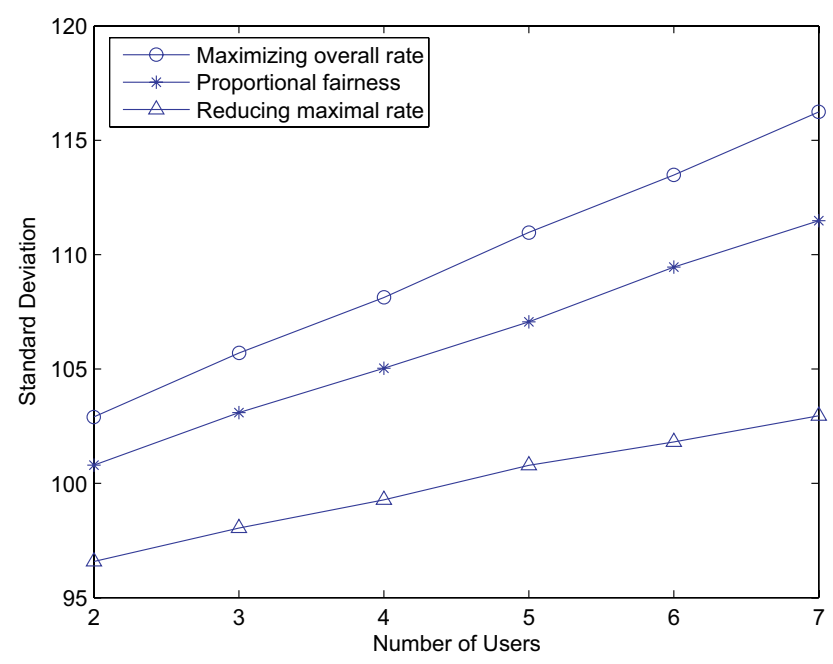

(b) Standard deviation vs. number of users

Fig. 6. Average rate and standard deviation of multiple-user system.

limited subbands with good channel conditions. Comparing the performances of three approaches, we can see that the proportional fairness yields slightly lower average rate than that of the maximizing overall rate approach, and both proportional fairness and maximizing overall rate approaches achieve much higher rates than that of the reducing maximal rate approach.

In Fig. 6(b), we show the standard deviations of the data rates of the users for three approaches. Here the standard deviation represents the fairness of allocation among users. We can observe that the standard deviation for every scheme increases with the number of users since the larger the number of users, the higher the variation of the rates. At any fixed number of user, the reducing maximal rate approach results in smallest standard deviation, and its standard deviation slightly increases with the number of users. This is because the feasible rates obtained from the reducing maximal rate approach are close to each other. In contrast, the maximizing overall rate scheme can yield the feasible rates of around 100 to 480 Mbps at the same time. Thus, its standard deviation increases much faster with the number of users. The standard deviation of proportional fairness approach is between the other two schemes. So the proportional fairness approach is a tradeoff between the maximal rate approach and reducing maximal rate approach for both performances and fairness.

\section{CONCLUSIONS}

Low power consumption is one of the key elements to make multiband UWB technology to be the solution for future indoor wireless communications. We propose in this paper an efficient cross layer algorithm for allocating subband and power among users in a multiband UWB system. The proposed scheme aims to reduce power consumption without compromising performance. We propose a general framework to minimize the overall transmit power under the practical implementation constraints. The formulated problem is $N P$ hard; however, with the proposed fast suboptimal algorithm, we can reduce the computational complexity to only $O\left(K^{2} S\right)$, where $K$ is the number of users and $S$ is the number of subbands. Simulation results show that the proposed algorithm achieves comparable performances to those of expensive optimal full search algorithm, and can save up to $61 \%$ of power consumption compared to the standard multiband scheme. Moreover, the proposed algorithm can obtain the feasible solutions adaptively when the initial system is not feasible for the rate requirements of the users. Among three different system optimization goals used in the proposed rate adaptation algorithm, the proportional fairness approach turns out to be a tradeoff between the maximal rate approach and reducing maximal rate approach for both performances and fairness.

\section{REFERENCES}

[1] Federal Communications Commission Report FCC 98-153, "Revision of Part 15 of the Commission's Rules Regarding Ultra-Wideband Transmission Systems, First Report and Order," Feb. 14, 2002.

[2] M. Z. Win and R. A. Scholtz, "Impulse radio: how it works," IEEE Commun. Letters, vol. 2, no. 2, pp. 36-38, Feb. 1998.

[3] J. R. Foerster, "The performance of a direct-sequence spread ultra wideband system in the presence of multipath, narrowband interference, and multiuser interference," inProc. IEEE Conf. on Ultra Wideband Systems and Technologies 2002, pp. 87-91.

[4] Z. Feng and T. Kaiser, "On channel capacity of multi-antenna UWB indoor wireless systems," in Proc. IEEE Int. Symposium on Spread Spectrum Techniques and Applications 2004.

[5] J. R. Foerster, et al., "Intel CFP presentation for a UWB PHY," IEEE P802.15-03/109r1, Mar. 2003.

[6] E. Saberinia and A. H. Tewfik, "Multi-user UWB-OFDM communications," in IEEE Proc. Pacific Rim Conf. on Commun., Computers and Signal Processing 2003, vol. 1, pp. 127-130.

[7] A. Batra, et al., "Design of a multiband OFDM system for realistic UWB channel environments," IEEE Trans. Microw. Theory Tech., vol. 52, no. 9, pp. 2123-2138, Sept. 2004.

[8] IEEE 802.15WPAN High Rate Alternative PHY Task Group 3a (TG3a). Internet: www.ieee802.org/15/pub/TG3a.html

[9] W. Zhuang, X. Shen, and Q. Bi, "Ultra-wideband wireless communications," Wireless Commun. and Mobile Computing, special issue on ultra-broadband wireless communications for the future (invited paper), vol. 3, no. 6, pp. 663-685, 2003.

[10] F. Cuomo, C. Martello, A. Baiocchi, and F. Capriotti, "Radio resource sharing for ad hoc networking with UWB," IEEE J. Sel. Areas Commun., vol. 20, no. 9, pp. 1722-1732, Dec. 2002.

[11] B. Radunovic and J.-Y. Le Boudec, "Optimal power control, scheduling, and routing in UWB networks," IEEE J. Sel. Areas Commun., vol. 22, no. 7, pp. 1252-1270, Sept. 2004.

[12] E. Saberinia, J. Tang, A. H. Tewfik, and K. K. Parhi, "Design and implementation of multi-band pulsed-OFDM system for wireless personal area networks," in Proc. IEEE Int. Conf. on Commun. 2004, vol. 2, pp. 862-866, Jun. 20-24. 
[13] Y. Nakache, et al., "Low-complexity ultrawideband transceiver with compatibility to multiband-OFDM," technical report, Mitsubishi Electronic Research Laboratory. Internet: www.merl.com/reports/docs/TR2004-051.pdf.

[14] J. Foerster, et al., "Channel Modeling Sub-committee Report Final," IEEE802.15-02/490, Nov. 2003.

[15] A. A. M. Saleh and R. A. Valenzuela, "A statistical model for indoor multipath propagation," IEEE J. Sel. Areas Commun., vol. 5, no. 2, pp. 128-137, Feb. 1987.

[16] A. F. Molisch, J. R. Foerster, and M. Pendergrass, "Channel models for ultrawideband personal area networks," IEEE Wireless Commun., vol. 10, no. 6, pp. 14-21, Dec. 2003.

[17] W. P. Siriwongpairat, Z. Han, and K. J. R. Liu, "Energy-efficient channel allocation for multiuser multiband UWB system," in Proc. IEEE Wireless Commun. and Networking Conf. 2005, pp. 813-818.

[18] J. G. Proakis, Digital Communications, Fourth Edition. New York: McGraw-Hill, 2001.

[19] H. Kellerer, U. Pferschy, and D. Pisinger, Knapsack Problems. New York: Springer, 2004.

[20] C. Y. Wong, R. S. Cheng, K. B. Letaief, and R. D. Murch, "Multiuser OFDM with adaptive subcarrier, bit, and power allocation," IEEE J. Sel. Areas Commun., vol. 17, no. 10, pp. 1747-1758, Oct. 1999.

[21] F. Kelly, "Charging and rate control for elastic traffic," European Trans. Telecommun., vol. 8, no. 1, pp. 33-37, Jan. 1997.

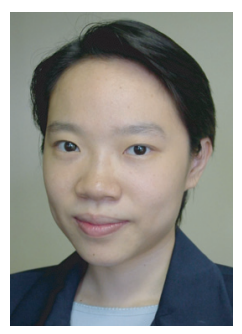

W. Pam Siriwongpairat (S'03-M'06) received the B.S. degree in electrical engineering from Chulalongkorn University, Bangkok, Thailand, in 1999, and the M.S. and Ph.D. degree both in electrical engineering from the University of Maryland, College Park, in 2001 and 2005, respectively. Her research interests span a broad range of areas from signal processing to wireless communications and networking, including space-time coding for multiantenna communications, cross layer design for wireless networks, communications in mobile ad hoc networks and wireless sensor networks, OFDM systems, and ultra-wideband communications.

Dr. Siriwongpairat is currently a wireless communications specialist with Meteor Communications Corporation working on research and development of wireless communications technology. From January 2005 to May 2005, she was a Postdoctoral Research Associate with the Department of Electrical and Computer Engineering and the Institute for Systems Research (ISR), University of Maryland, College Park.

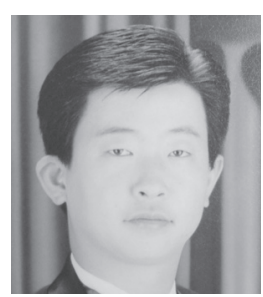

Zhu Han (S'01-M'04) received the B.S. degree in electronic engineering from Tsinghua University, in 1997, and the M.S. and Ph.D. degrees in electrical engineering from the University of Maryland, College Park, in 1999 and 2003, respectively.

From 2000 to 2002 , he is an R\&D Engineer of ACTERNA, Germantown, Maryland. From 2002 to 2003, he was a Graduate Research Assistant at the University of Maryland. From 2003 to 2006, he was a Research Associate at the University of Maryland. Currently, he is an assistant Professor in Electrical and Computer Engineering Department at Boise State University, Idaho, USA. His research interests include wireless resource allocation and management, wireless communications and networking, game theory, and wireless multimedia.

Dr. Han is a member of the Technical Programming Committee for the IEEE International Conference on Communications, the IEEE Vehicular Technology Conference, the IEEE Consumer Communications and Networking Conference, the IEEE Wireless Communications and Networking Conference, and the IEEE Globe Communication Conference.

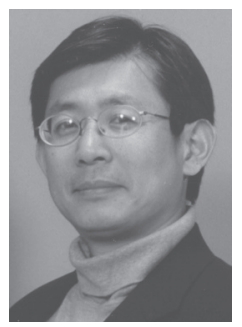

K. J. Ray Liu (F’03) is Professor and Associate Chair of Graduate Studies and Research of Electrical and Computer Engineering Department, University of Maryland, College Park. His research contributions encompass broad aspects of wireless communications and networking, information forensics and security, multimedia communications and signal processing, bioinformatics and biomedical imaging, and signal processing algorithms and architectures.

Dr. Liu is the recipient of numerous honors and awards including best paper awards from IEEE Signal Processing Society (twice), IEEE Vehicular Technology Society, and EURASIP; IEEE Signal Processing Society Distinguished Lecturer, EURASIP Meritorious Service Award, and National Science Foundation Young Investigator Award. He also received various teaching and research awards from University of Maryland including Poole and Kent Company Senior Faculty Teaching Award and Invention of the Year Award.

Dr. Liu is Vice President - Publications and on the Board of Governor of IEEE Signal Processing Society. He was the Editor-in-Chief of IEEE Signal Processing Magazine and the founding Editor-in-Chief of EURASIP Journal on Applied Signal Processing. 